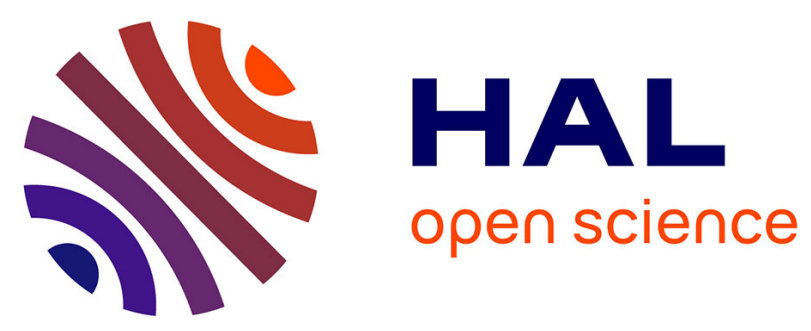

\title{
Oxidation behaviour of carbon monoxide at the photostimulated surface of $\mathrm{ZnO}$ nanowires
}

Ch y Wang, M Kinzer, S K Youn, N Ramgir, M Kunzer, K Köhler, M

Zacharias, V Cimalla

\section{- To cite this version:}

Ch y Wang, M Kinzer, S K Youn, N Ramgir, M Kunzer, et al.. Oxidation behaviour of carbon monoxide at the photostimulated surface of $\mathrm{ZnO}$ nanowires. Journal of Physics D: Applied Physics, 2011, 44 (30), pp.305302. 10.1088/0022-3727/44/30/305302 . hal-00637803

\section{HAL Id: hal-00637803 \\ https://hal.science/hal-00637803}

Submitted on 3 Nov 2011

HAL is a multi-disciplinary open access archive for the deposit and dissemination of scientific research documents, whether they are published or not. The documents may come from teaching and research institutions in France or abroad, or from public or private research centers.
L'archive ouverte pluridisciplinaire HAL, est destinée au dépôt et à la diffusion de documents scientifiques de niveau recherche, publiés ou non, émanant des établissements d'enseignement et de recherche français ou étrangers, des laboratoires publics ou privés. 
We are very thankful for the advises from the reviewers and the editor. The manuscript (Article ID: D/393066/PAP) has already been revised according to the comments (resubmitted online).

Following are the comments and the answers,

corresponding the second referee's report: "I find the paper to be well written,..., I recommend this paper for publication."

We appreciate your taking time to review the manuscript and your commendation.

corresponding the third referee's report:

Comment \#1: "In the Introduction, the authors should include some references dealing with room temperature sensors based on UV-activation of metal oxide nanowires..."

Answer \#1: Some references about the UV-activation of nanostructured materials have now been given in the introduction: "Thus, the photostimulated sensor, in particular based on nanostructured materials [15], enables gas detection at room temperature, resulting in a reduced power consumption [16]."

Comment \#2: "... why is there no particle seed formation on the bare Si in between the Au lines" Answer \#2: The growth mechanism has been shortly explained in the text and a literature has been given: "The self-assembled monolayers (SAMs) of MPA, possessing two functional groups, namely, thiol and carboxylic acid, were selectively formed on the gold lines and used to discriminate between Au and Si surface as well as to interact with cationic Zn species in the solution phase. The growth mechanism has been explained in our previous work [14]."

Comment \#3: "Why is there no Au-supported growth on the metal lines without the particle layer at the high temperatures used. Can it be ruled out"

Answer \#3: The $\mathrm{ZnO}$ nanowires have been grown based not on the vapour-liquid-solid (VLS) but on the vapour-solid (VS) principle. Furthermore, we have not found any Au particles on the top of the $\mathrm{ZnO}$ nanowires.

Comment \#4: "Authors should provide information how the electrical responses are extracted..." Answer \#4: The reviewer is right. The information of the electrical measurements is missing. Two sentences have been added in the experimental section: "Two electrical contacts were brought onto the two outer Au-lines. The electrical resistance of the $\mathrm{ZnO}$ nanowires between the Au-lines were determined during the sensing experiment, while the UV LED was regularly switched on and off for photostimulation and CO oxidation, respectively."

Comment \#5: "page 8 line 4 absorbed should read as adsorbed"

Answer \#5: The word in the text has been changed to be "adsorbed".

Comment \#6: “The non-equilibrium conditions with desorption of gaseous species at low pressure and inert gases are not close to realistic conditions in the environment. The authors should comment how this technique can lead to an operating room temperature sensor as stated in the manuscript"

Answer \#6: That's totally right. The measuring conditions described in this work are not close to normal realistic conditions in the environment which contains high concentration oxygen/water vapor as main interfering gases. Thus, we proposed in the conclusion that such sensors working at room temperature will first be easy to be used for special applications, such as for $\mathrm{CO}$ detection in fuel cells where $\mathrm{H} 2$ is the main gas. For $\mathrm{CO}$ detection in $\mathrm{H} 2 \mathrm{O}$ and $\mathrm{O} 2$ containing environment, the response of $\mathrm{H} 2 \mathrm{O}$ and $\mathrm{O} 2$ must first be determined for the calculation of pure $\mathrm{CO}$ response.

However, further experiments will be needed for such applications. 
Comment \#7: "Authors should include the dipole nature of the $\mathrm{CO}$ molecule and the two potential interaction modes with the surface..."

Answer \#7: We have now proposed one possible mechanism of $\mathrm{CO}$ molecules adsorbed on the nanowire surface at the end of $3.3 \mathrm{CO}$ sensing mechanism, and mentioned "However, further experiments are still desired to understand the dipole nature of the CO molecules adsorbed on the surface.".

Sincerely yours,

Chunyu Wang 


\title{
Oxidation behavior of carbon monoxide at photostimulated surface
}

\section{of $\mathrm{ZnO}$ nanowires}

\author{
Ch Y Wang', M Kinzer ${ }^{1}$, S K Youn ${ }^{2}$, N Ramgir ${ }^{2}$, M Kunzer ${ }^{1}$, K Köhler ${ }^{1}$, M Zacharias ${ }^{2}$ and V \\ Cimalla $^{1}$
}

${ }^{1}$ Fraunhofer-Institute for Applied Solid-State Physics (IAF), D-79108 Freiburg, Germany

${ }^{2}$ Department of Microsystems Engineering, University of Freiburg, 79110 Freiburg, Germany

E-mail: chunyu.wang@iaf.fraunhofer.de

Abstract. Normally carbon monoxide is considered to be a reducing agent, which can be used for CO detection. However, oxidizing behavior of $\mathrm{CO}$, to the best of our knowledge, has not been reported. In this work, we find that CO can also act as oxidizing gas at room temperature on photostimulated $\mathrm{ZnO}$ nanowires. For photostimulation, the low intensity of light emitting diodes a is used to induce a very low photo current, which, however, leads to a large resistance change due to the ultraviolet light interaction with the nanowire surface. During CO detection, the oxygen vacancies enhance the CO adsorption on the nanowire surface even at room temperature. $\mathrm{CO}$ molecules adsorbing on the surface are presumably bound to a zinc oxygen vacancy complex, causing a reduction of free electron concentration, which leads to an oxidizing effect of CO gas. An adsorption model for $\mathrm{CO}$ is proposed to explain the experimental observations. These results shed light on developing room-temperature $\mathrm{CO}$ sensor using oxide nanostructures with the help of high energetic photon replacing the high temperature process normally used in traditional gas sensors.

Keywords $\mathrm{ZnO}$ nanowires, photon stimulation, $\mathrm{CO}$ sensing, oxidation behavior, photoluminescence

\section{Introduction}

In recent years, nanotechnology research has focused on the understanding of physical properties of nanomaterials and their consequent applications [1], e. g. for gas detection. Metal oxide nanostructures have been demonstrated to be highly sensitive to $\mathrm{CO}$ [2], $\mathrm{NH}_{3}$ [3], $\mathrm{H}_{2}$ [4], $\mathrm{NO}_{\mathrm{x}}$ [5], as well as to ethanol [6]. Among 
them, the detection of $\mathrm{CO}$ as one of the major pollutants in the automobile exhaust gases has attracted much interest. Carbon monoxide, normally considered to be a reducing gas [7], can be detected at high temperatures using metal oxides as gas sensing material such as $\mathrm{ZnO}$ [8], $\mathrm{TiO}_{2}$ [9], $\mathrm{NiO}$ [10] and $\mathrm{CuO}$ [11]. The sensing mechanisms are, however, not fully understood.

For the environmental sensing behavior, two models are used to explain the interaction of $\mathrm{CO}$ with a metal oxide surface and adsorbed species. The first model assumes that oxygen molecules adsorb on the surface of metal oxides in a form of $\mathrm{O}^{2-}$. When $\mathrm{CO}$ is present with $\mathrm{O}^{2-}$ at high temperature, the reaction $\mathrm{CO}+\mathrm{O}^{2-} \rightarrow \mathrm{CO}_{2}+2 \mathrm{e}^{-}$proceeds on the surface, leading to a release of localized electrons [12]. According to the second model, oxygen adsorbs as $\mathrm{O}_{2}^{-}$. At low temperature, $\mathrm{CO}$ molecules do not have sufficient thermal energy to react with $\mathrm{O}_{2}^{-}$, while at a temperature above $300^{\circ} \mathrm{CO}_{2}^{-}$is converted to $\mathrm{O}^{-}$and $\mathrm{CO}$ reacts via $\mathrm{CO}+\mathrm{O}^{-} \rightarrow \mathrm{CO}_{2}+\mathrm{e}^{-}$. In both cases the detection scheme for $\mathrm{CO}$ involves the reaction with pre-adsorbed oxygen, and normal $\mathrm{CO}$ sensors based on metal oxides work only at high temperature $\left(>300^{\circ} \mathrm{C}\right)$ using its reducing effect $[7,13]$. However, to the best of our knowledge, oxidizing behavior of $\mathrm{CO}$ has not been reported.

In our previous work, we enabled the surface reaction of $\mathrm{CO}$ at room temperature by photostimulation [14]. Here, the high energetic photon replaces the high temperature process normally used in high-temperature gas sensors. Thus, the photostimulated sensor, in particular based on nanostructured materials [15], enables gas detection at room temperature, resulting in a reduced power consumption [16]. The gas sensing mechanism stays, however, still controversial due to the interference of remaining residual gases with photostimulated surface. In this work, the effect of photon stimulation on the nanowire surface has been studied at well-defined conditions. Furthermore, experiments at alternating measuring conditions have been carried out to extract a pure sensing signal. As a result, the oxidizing character of $\mathrm{CO}$ on the photostimulated surface of $\mathrm{ZnO}$ nanowires and the related sensing behavior have been exhibited. The pure $\mathrm{CO}$ response has been calculated based on a simple gas adsorption model. The mechanism of $\mathrm{CO}$ adsorption at the photostimulated surface has been proposed.

\section{Experimental Section}

The $\mathrm{ZnO}$ nanowires were grown using the vapor-solid (VS) method [14] and characterized by highresolution scanning electron microscopy (SEM, Nova NanoSEM, FEI). First, the Si substrate was patterned with Au electrodes by conventional photolithography, physical vapor deposition (Au: $100 \mathrm{~nm}, \mathrm{Cr}: 5 \mathrm{~nm}$ ) and lift off processing, followed by surface modification of Au by immersing the substrates into a $1 \mathrm{mM}$ ethanol solution of 
2-mercaptopropionic acid, MPA (Sigma Aldrich, 99.8\%) for $5 \mathrm{~h}$ and subsequent washing with ethanol to remove excess MPA. The self-assembled monolayers (SAMs) of MPA, possessing two functional groups, namely, thiol and carboxylic acid, were selectively formed on the gold lines and used to discriminate between $\mathrm{Au}$ and $\mathrm{Si}$ surface as well as to interact with cationic $\mathrm{Zn}$ species in the solution phase. The growth mechanism has been explained in our previous work [14]. The distance between two Au lines was $2 \mu \mathrm{m}$. After that, zinc acetate dihydrate (Sigma Aldrich, 98\%) in methanol was dropped onto the substrates, followed by drying in air at $70^{\circ} \mathrm{C}$ and annealing at $350^{\circ} \mathrm{C}$ for $20 \mathrm{~min}$ in air, which resulted in $\mathrm{ZnO}$ seeds. Finally, $\mathrm{ZnO}$ nanowire arrays were grown at $930^{\circ} \mathrm{C}$ by vapor-phase deposition using powdered $\mathrm{ZnO}$ (Sigma Aldrich, 99.9\%) and graphite (Alfa Aesar, 99.9\%) as a source material and $\mathrm{O}_{2}$ as an oxidizing agent.

The CO sensing experiments were conducted in a custom-built equipment [16]. The $\mathrm{ZnO}$ nanowires were placed in the measuring chamber with an ultraviolet light emitting diode (UV LED with $\lambda \sim 356 \mathrm{~nm}$ and optical power $\sim 200 \mu \mathrm{W}$ ) mounted above the sample. Two electrical contacts were brought onto the two outer Au-lines. The electrical resistance of the $\mathrm{ZnO}$ nanowires between the Au-lines were determined during the sensing experiment, while the UV LED was regularly switched on and off for photostimulation and CO oxidation, respectively. To understand the influence of photostimulation on $\mathrm{ZnO}$ nanowires, photoluminescence measurements were carried out using different excitation sources.

\section{Results and Discussion}

\subsection{Optical Properties of ZnO Nanowire Arrays}

Figure 1 shows a SEM image of $\mathrm{ZnO}$ nanowire arrays grown on an Au-patterned $\mathrm{Si}$ substrate. Mechanical contacts between nanowires grown on two neighboring Au patterns are forming nanowire bridges as shown schematically in the inset of Fig. 1. The abundant nanowire bridges establish a current flow between nanowires deposited on separated neighboring Au electrodes. Furthermore, due to the increased surface area of such arrays of $\mathrm{ZnO}$ nanowires, the gas sensing properties are expected to be enhanced. The structural properties of the as grown $\mathrm{ZnO}$ nanowire arrays have been reported elsewhere [17]. 


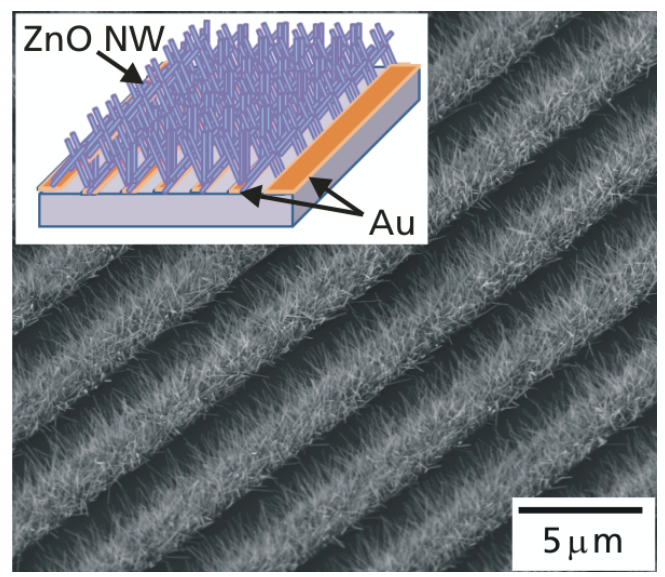

Figure 1. SEM image taken at $45^{\circ}$ tilt of $\mathrm{ZnO}$ nanowires grown on an Au-patterned Si substrate. The inset shows a schematic view of $\mathrm{ZnO}$ nanowire bridges.

The grown $\mathrm{ZnO}$ nanowires were first investigated by photoluminescence (PL) using different excitation wavelengths and varied excitation intensities. The results are shown in Fig. 2. The four curves (Fig. 2, solid lines) were obtained using a $\mathrm{HeCd}$ laser $(\lambda \sim 325 \mathrm{~nm}, h v \sim 3.81 \mathrm{eV})$ at different power densities from 0.01 to 10 $\mathrm{W} / \mathrm{mm}^{2}$. The excitation area was about $10^{-3} \mathrm{~mm}^{2}$. A relatively sharp main peak appears at a photon energy of $3.29 \mathrm{eV}$, which is in agreement with the reported value in literature [18]. This UV emission is normally attributed to the strong band-edge emission [18]. A broad emission peak centered at $\sim 2.45 \mathrm{eV}$ can also be found in Fig. 2 . The origin of this green emission is still under discussion. Very often the green emission has been attributed to defects on the surface of $\mathrm{ZnO}$ [18], considering singly ionized oxygen vacancies [19], zinc interstitials [20], zinc vacancies [21], as well as antisite defects [22]. 

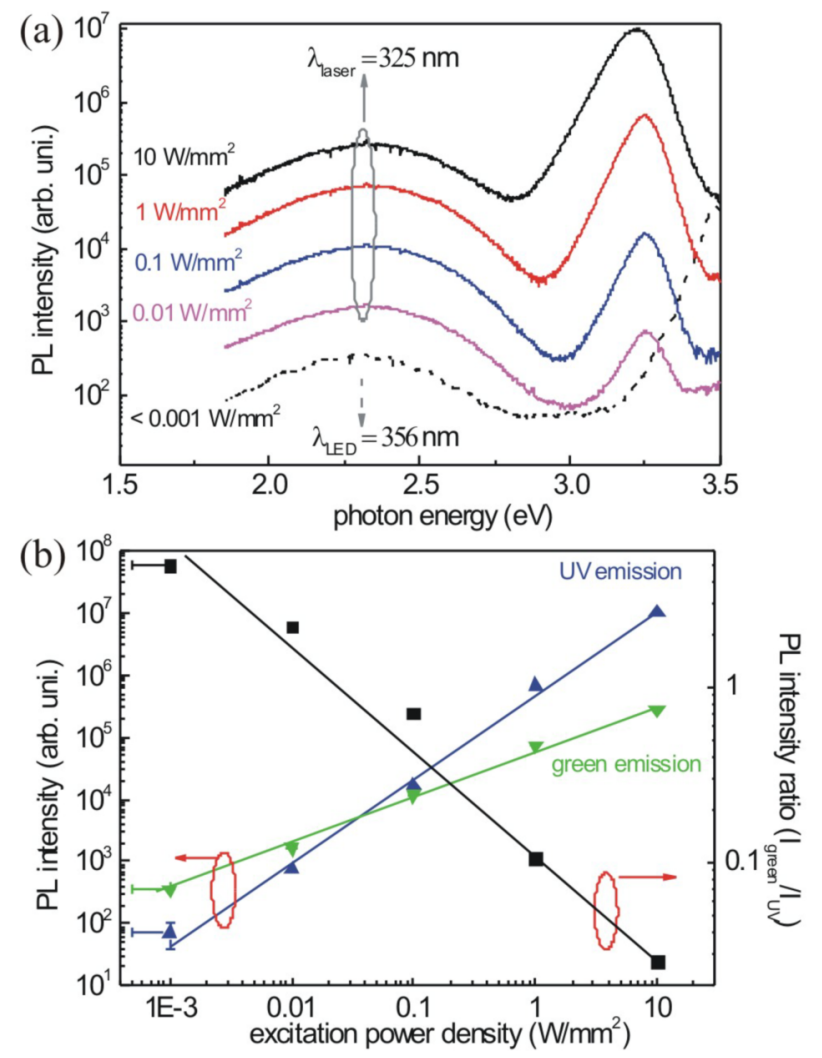

Figure 2. (a) PL measurements using different exciting sources and excitation power densities: solid lines represent the excitation by a HeCd laser $(\lambda \sim 325 \mathrm{~nm})$ and dashed lines by a UV LED $(\lambda \sim 356 \mathrm{~nm})$; (b) PL intensity and intensity ratio between the green and UV emission as a function of excitation power density.

The intensities of both UV and green emission have been found to be dependent on the excitation power density. In case of our $\mathrm{ZnO}$ nanowire arrays, both the intensities of the UV and green emission increase by increasing the excitation power density (Fig. 2 (b)). Note that the measurements integrate over a large number of nanowires and are not done on single wires. No saturation effect was seen for both PL contributions. The ratio of the PL intensities $\left(I_{\text {green }} / I_{\mathrm{UV}}\right)$ decreases with the increase of the excitation power density.

Normally, the UV band edge emission peak increases by increasing the excitation intensity as reported for instance by Shi et al. [23]. In contrast, the intensity of the green emission of high-quality ZnO films grown by metal organic chemical vapor deposition is reported to saturate when increasing the excitation intensity. This effect has been attributed to the perfect crystal structure and the very low number of surface states in single crystalline $\mathrm{ZnO}$ films [24]. In the case of $\mathrm{ZnO}$ nanostructures, the intensity of green emission was found to increase with the increasing excitation intensity and no saturation was observed [23], which agrees well with our observations as can be seen in Fig. 2 (b). The opposite behavior of the green luminescence in epitaxial films and 
in nanowires strongly supports the assumption that surface states are the main source for these differences. Following the interpretation of Ahn et al. [25], oxygen vacancies are assumed to be the major source for these surface defect states. As discussed before, these vacancies are the active centers in the $\mathrm{CO}$ adsorption on $\mathrm{ZnO}$ and, thus, are also responsible for their sensing mechanism [26,27].

The same PL experiment was carried out by replacing the excitation source HeCd laser with a UV LED $(\lambda \sim 356 \mathrm{~nm})$, which was also used for photostimulation of $\mathrm{ZnO}$ nanowires and which has a much lower power intensity than the laser source. The overall power density of the UV light irradiating the $\mathrm{ZnO}$ nanowires was much lower than $1 \mathrm{~mW} / \mathrm{mm}^{2}$. The respective PL spectrum is the dashed-line curve in Fig. 2 (a) representing the signal for a 5 min excitation with the UV LED. The main peak centered at $\sim 3.48 \mathrm{eV}$ is due to the $356 \mathrm{~nm} \mathrm{LED}$ excitation, which is not completely blocked in the system. The band gap emission from the $\mathrm{ZnO}$ nanowires is negligible for such weak excitation. The observed green luminescence is located at the same energy position as observed if using the $\mathrm{HeCd}$ laser as excitation source (Fig. 2 (a)). Although the excitation power is very low, the intensity of the green luminescence is still relatively high indicating the existence and easy excitation of related defects at the surface of $\mathrm{ZnO}$ nanowires with its large surface area. The PL experiments only cannot clarify if these defects are pre-existent or have been photostimulated by the UV illumination. Nevertheless, it suggests that the nanowires have a high number of photon active centers at the surface, which are also expected to be responsible for gas sensing, thus, making nanowires ideal candidates for sensing applications.

\subsection{Oxidizing behavior of carbon monoxide at Photostimulated $\mathrm{ZnO}$ Nanowire Surface}

The $\mathrm{ZnO}$ nanowires were further investigated with respect to $\mathrm{CO}$ sensing character at room temperature and the influence of photostimulation. Two main steps are involved in the $\mathrm{CO}$ sensing process: (i) gas adsorption at the nanowire surface during $\mathrm{CO}$ exposure and (ii) reactivation of the surface via UV illumination. To understand the $\mathrm{CO}$ sensing behavior at photostimulated surface, the following four effects have to be considered: (I) photo current, (II) gas desorption during photostimulation, (III) surface adsorption of CO gas, and (IV) a competing surface adsorption of ambient residual gases $\left(\mathrm{H}_{2} \mathrm{O}\right.$ and $\left.\mathrm{O}_{2}\right)$ from the measuring chamber during the $\mathrm{CO}$ adsorption process [14]. In our previous work, a large resistance change has been observed after photostimulation and $\mathrm{CO}$ exposition, which is preliminary assumed to be caused by an interaction between $\mathrm{CO}$ and residual water vapor / oxygen molecules at the nanowire surface. In the following, the four main processes 
are studied to exclude the influence of the residual gases remaining in the measuring system and to understand the interaction between pure $\mathrm{CO}$ gas and the surface of photostimulated nanowires.

First, photocurrent has been determined with the help of electrical measurements in different processes, as shown in Figure 3. The black curve represents the resistance change by periodically switching the UV LED “on" (photostimulation) and "off" for 2 hours with a continuous $\mathrm{N}_{2}$ purge of the system. It must be mentioned here that the gas adsorption/desorption process at room temperature is time-consuming due to the low thermal energy of the gas molecules. Thus, resistance has been measured at non-equilibrium state, i. e. no saturation value of resistance was reached, which, however, has already been proven to be an efficient method to obtain sensing signals, in particular for photostimulated gas sensors operated at room temperature [28]. During photostimulation, the system was pumped down to $\sim 10^{-3}$ mbar, while the system was filled with $\mathrm{N}_{2}$ after switching off the UV LED (black). Theoretically, a large photocurrent or a large resistance change due to photogenerated carriers should be induced by switching on or off the above-band-gap excitation $\left(\mathrm{E}_{\mathrm{ZnO}}=3.37 \mathrm{eV}\right)$ [29]. As can be seen from the black curve, the resistance change after first photostimulation is nearly two orders of magnitude, then decreased only slightly for the second and third time irradiation with UV light. In particular, it was observed that the resistance increased only insignificantly after switching off the UV light. The same experiments were carried out with different UV LEDs (i.e. $h v=3.26$ and $4 \mathrm{eV}$, respectively), and similar resistance changes were obtained independent of the used energy. A very low photocurrent was observed after photostimulation using a UV-LED with photon energy well above the band gap of $\mathrm{ZnO}$ (4 eV). This agrees very well with the PL characterization of $\mathrm{ZnO}$ nanowire arrays, which has shown a negligible band gap emission using the UV LED as excitation source.

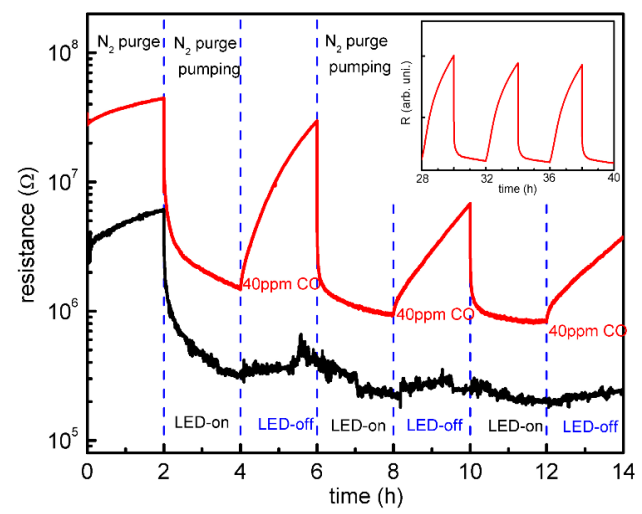

Figure 3. Resistance change of the $\mathrm{ZnO}$ nanowires under photostimulation (black) and photostimulation/CO exposure (red). The inset shows the resistance change after four cycles of photostimulation/CO exposure. 
The observed large resistance decrease after the first UV-light illumination can be interpreted by a charge transfer at surface defects. According to photoelectron spectroscopy analysis [26,27], these defects are oxygen vacancies. This indicates that the defects observed in the former PL experiments are generated by the UV illumination. In an equilibrium state before the first photoexcitation, adsorbates from environment preferable bond to $\mathrm{Zn}$ ions at these oxygen vacancies resulting in a localized negative charge. The UV-light forces desorption of these charged adsorbates, which in turn results in the generation of free electrons and, thus, in an enhanced surface carrier transport. The PL measurements indeed showed the green luminescence observed even at low excitation power densities, hence indicating a high number of optically active centers at the nanowire surface. However, after the first photoexcitation, the oxygen vacancies can only be compensated very slowly by the residual water vapor / oxygen residual gases in the $\mathrm{N}_{2}$ environment of the experimental setup, leading to a slight resistance increase in repeating LED-off procedures. In contrast, a clear electrical response was observed on $\mathrm{ZnO}$ nanowires after repeated photoexcitation cycles in $\mathrm{CO}$ with concentrations as low as $40 \mathrm{ppm}$ in $\mathrm{N}_{2}$ (red curve in Fig. 3). As can be seen in Fig. 3, however, the resistance change decreases gradually with the number of the photostimulation/CO exposure cycles. After 6 cycles, a nearly constant resistance change to the photostimulation/CO exposure cycle was observed as demonstrated in the inset of Fig. 3. Although it has already been reported that the residual gases, such as oxygen or humidity, can oxidize $\mathrm{ZnO}$ surface, in particular react with oxygen vacancies even under ultrahigh vacuum conditions [30], this experiment indicates evidently that the $\mathrm{CO}$ molecules has obviously been adsorbed on the photostimulated surface at room temperature and has large influence on the electrical properties of $\mathrm{ZnO}$ nanowires. Furthermore, the large resistance increase after $\mathrm{CO}$ exposure signifies an oxidizing behavior of adsorbed $\mathrm{CO}$ molecules at the surface of photostimulated $\mathrm{ZnO}$ nanowires.

To determine the influence of residual gas adsorption in the chamber, such as $\mathrm{H}_{2} \mathrm{O}$ and $\mathrm{O}_{2}$, the $\mathrm{ZnO}$ nanowires were first exposed to $40 \mathrm{ppm} \mathrm{CO}$ in $\mathrm{N}_{2}$ together with the residual gases. Then, the chamber was evacuated down to $10^{-3}$ mbar and purged with $\mathrm{N}_{2}$ to reduce the residual gas concentration. This process was repeated several times. The corresponding resistance change is shown in Fig. 4 (a). Again, the resistance strongly decreases after the first UV irradiation due to desorption of charged species from oxygen vacancies and then increases only slightly after residual gas exposure (mainly $\mathrm{H}_{2} \mathrm{O}$ ). After exposure to $40 \mathrm{ppm} \mathrm{CO}$ and residual gases, the $\mathrm{ZnO}$ nanowires show a much higher resistance change in comparison to the exposure to residual gases 
alone, indicating an oxidizing behavior of the $\mathrm{CO}$ gas. A summary of the responses towards different gases is shown in Fig. 4 (b). The sensing process involves adsorption of different gas molecules (CO and residual gases) at the photostimulated $\mathrm{ZnO}$ surface. Under the assumption according to the Langmuir adsorption isotherm that the surface adsorption sites are equivalent, and the adsorbed molecules do not interact, the pure CO response can be separated from the response to residual gases.
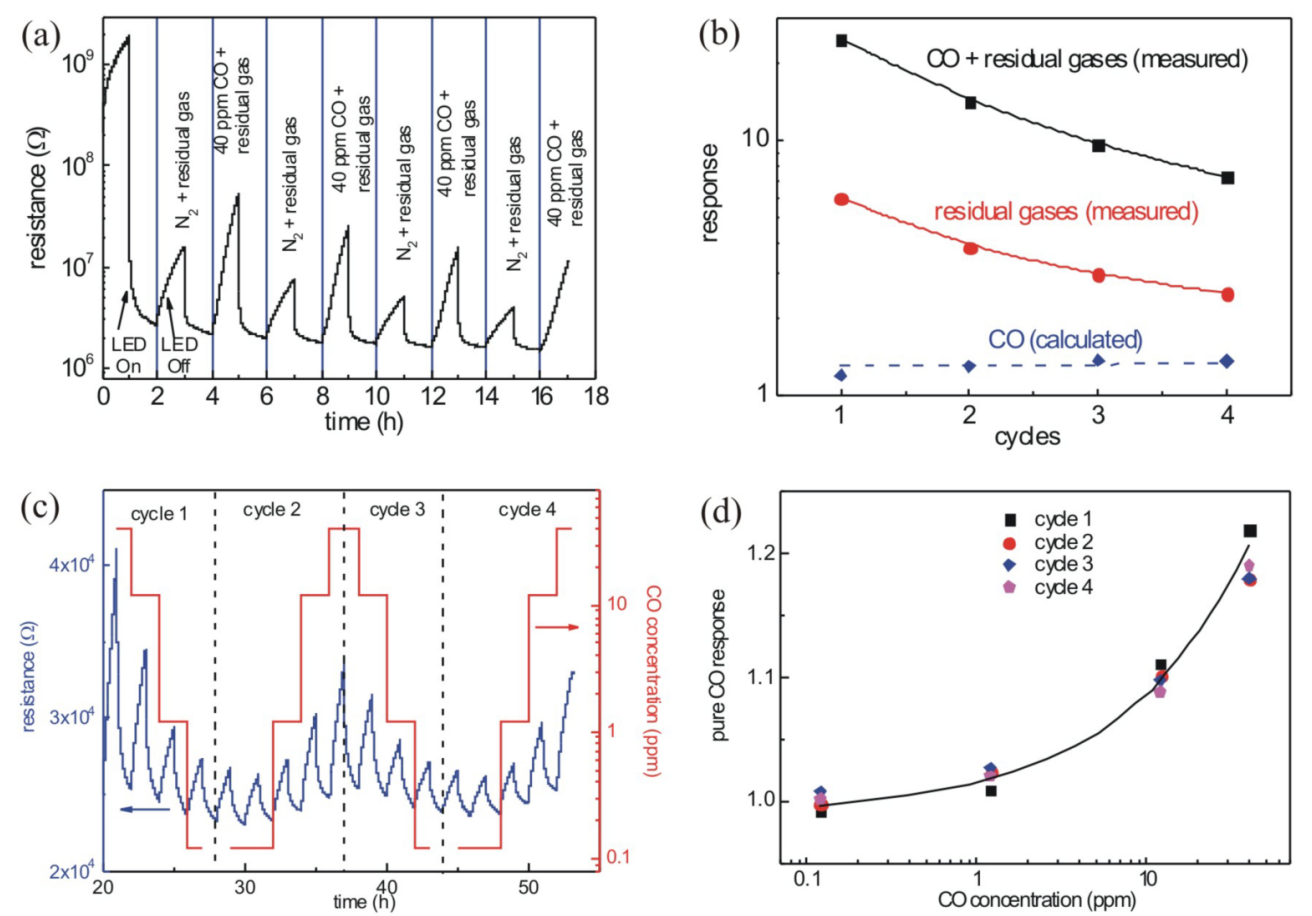

Figure 4. (a) Resistance change of $\mathrm{ZnO}$ nanowires periodically exposed to residual gases (mainly $\mathrm{H}_{2} \mathrm{O}$ and $\mathrm{O}_{2}$ ) and 40 ppm CO in $\mathrm{N}_{2}$; (b) measured nanowire response from (a) and calculated response towards $40 \mathrm{ppm} \mathrm{CO}$ in dependence on the photostimulation/oxidation cycles, (c) resistance change of $\mathrm{ZnO}$ nanowires in $\mathrm{CO}$ with different concentration, and (d) $\mathrm{ZnO}$ nanowire response in dependence on the $\mathrm{CO}$ concentration.

The response towards residual gases (red curve in Fig. 4 (c)) is defined as

$$
r e s p_{\mathrm{res}}=\frac{R_{\mathrm{res}}}{R_{\mathrm{UV}}}=\frac{S_{U V}}{S_{\text {res }}},
$$

where $R_{\text {res }}\left(S_{\text {res }}\right)$ and $R_{\mathrm{UV}}\left(S_{U V}\right)$ are the resistance (conductance) of $\mathrm{ZnO}$ nanowires after residual gas exposure and UV illumination, respectively, which decreases (increases) with the increase of the photostimulation/oxidation cycle number. This is caused by a reduction of the concentration of residual gases in the system by repeated 
purging of the chamber with $\mathrm{N}_{2}$ and pumping the chamber down to $\sim 10^{-3}$ mbar after every gas exposure. Accordingly, the response towards 40 ppm CO and residual gases (black curve in Fig. 4 (b)) is defined as:

resp $_{\text {Co\&res }}=\frac{R_{\mathrm{CO \&} \text { res }}}{R_{\mathrm{UV}}}=\frac{S_{U V}}{S_{\text {Co\&res }}}$,

where $R_{\mathrm{CO} \text { \&res }}\left(S_{\text {CO\&res }}\right)$ is the resistance (conductance) of $\mathrm{ZnO}$ nanowires after exposure to $\mathrm{CO}$ and residual gases, which decreases (increases) similarly but not identically with (1). Furthermore, as can be seen in Fig. 4 (b), the behavior of the two response curves (black and red curves) is very similar, indicating that the decreasing response to $\mathrm{CO}$ and residual gases in dependence of the measuring cycle was mainly caused by the reduction of the residual gas concentration. Similar to the definition of responses resp $p_{\text {res }}$ and resp co\&res $_{\text {, }}$, the pure CO response is defined as:

$\operatorname{resp}_{C O}=\frac{R_{C O}}{R_{U V}}=\frac{S_{U V}}{S_{C O}}$

where $R_{\mathrm{CO}}\left(S_{C O}\right)$ is the resistance (conductance) of $\mathrm{ZnO}$ nanowires after exposure to pure $40 \mathrm{ppm} \mathrm{CO}$. Due to the coexistence of $\mathrm{CO}$ and residual gases during the whole measurement, the resistance of $\mathrm{ZnO}$ nanowires after pure $\mathrm{CO}$ exposure $\left(R_{C O}\right)$ and the pure $\mathrm{CO}$ response defined in (3) cannot be directly determined from the resistance change curve shown in Fig. 4 (a). Hence, in this work, a simple model is used to calculate the pure CO response from the other two responses taking into account the resistance change due to exposure to different gases.

For the relatively small measured changes of the resistivity in Fig. 4 (a), the electron mobility is considered to be constant. Then, the resistance and conductance of the nanowires can be expressed by:

$S=X \cdot n$,

where $\mathrm{X}$ is a constant and $\mathrm{n}$ the electron concentration. The constant value $\mathrm{X}$ is expressed by $X=e \mu A / l$, where $e$ and $\mu$ are the electron charge and mobility, respectively, $l$ the nanowire length, and $A$ the nanowire cross section area. Thus, the nanowire conductance after photostimulation can be described using the simplified equation:

$S_{U V}=n_{\max } \cdot X$

where $n_{\max }$ is the maximum electron density in the nanowires after photostimulation. For the response on gases, it is assumed that different gas species occupy individual adsorption sites [31] and that at the low partial pressures used here these adsorption sites are not affecting each other. Furthermore, from the resistance increase, the 
oxidizing behavior of $\mathrm{CO}$ and the residual gases $\left(\mathrm{O}_{2}\right.$ or $\left.\mathrm{H}_{2} \mathrm{O}\right)$ can be concluded, thus, every adsorbed molecule will be negatively charged by localizing one or more electrons. As a consequence, the electron concentration is reduced by $\Delta n_{C O}$ or $\Delta n_{\text {res }}$ after exposure to pure $\mathrm{CO}$ or residual gases, respectively. Thereby, the conductance of the $\mathrm{ZnO}$ nanowires after gas exposure can be calculated by:

$$
\begin{aligned}
& S_{C O}=\left(n_{\max }-\Delta n_{C O}\right) \cdot X, \\
& S_{\text {res }}=\left(n_{\max }-\Delta n_{\text {res }}\right) \cdot X, \\
& S_{\text {CO\& res }}=\left(n_{\text {max }}-\Delta n_{C O}-\Delta n_{\text {res }}\right) \cdot X .
\end{aligned}
$$

Based on eq. (1) - (8), the pure CO response is calculated by:

$$
\frac{1}{r e s p_{C O}}=1+\frac{1}{\text { resp }_{\text {Co\&res }}}-\frac{1}{\text { resp res }}
$$

This calculated pure CO response (Fig. 4 (b), blue-dashed curve), remains nearly constant during the whole measurements, indicating that $\mathrm{CO}$ gas has a stable oxidizing effect at the photostimulated $\mathrm{ZnO}$ nanowire surface. Furthermore, the resistance change during $\mathrm{CO}$ and residual gas exposure is found to be clearly faster than that during exposure to residual gases alone, caused by the higher $\mathrm{CO}$ concentration ( $40 \mathrm{ppm}$ ) compared to the concentration of residual $\mathrm{H}_{2} \mathrm{O}$ or $\mathrm{O}_{2}(<1 \mathrm{ppm})$. These experiments clearly prove that pure $\mathrm{CO}$ has an oxidizing effect at room temperature if the surface of the $\mathrm{ZnO}$ nanowires has been photostimulated by UV light.

Based on the results determined by equation (9) it can be concluded that for a fixed $\mathrm{CO}$ flux the $\mathrm{ZnO}$ nanowires have a constant response. Next, $\mathrm{ZnO}$ nanowires were exposed to $\mathrm{CO}$ with different concentrations in $\mathrm{N}_{2}$ environment using the same measuring conditions. The measuring chamber was first purged with $\mathrm{N}_{2}$ for $20 \mathrm{~h}$ to reduce the concentration of residual gases. Then the $\mathrm{ZnO}$ nanowires were photostimulated for $1 \mathrm{~h}$, followed by $\mathrm{CO}$ oxidation for $1 \mathrm{~h}$ (Fig. 4 (c). The procedure was repeated by varying the $\mathrm{CO}$ concentration from 0.12 to 40 ppm, as shown in cycle 1 in Fig. 4 (c). As expected for oxidizing behavior, the resistance change decreases with decreasing $\mathrm{CO}$ concentration in cycle 1 . To study the reproducibility of the $\mathrm{CO}$ sensing behavior at the photon stimulated $\mathrm{ZnO}$ nanowire surface, measurements with increasing $\mathrm{CO}$ concentration (cycle 3) as well as with decreasing CO concentration from 40 to 0.12 ppm (cycle 2 and 4 in Fig. 4 (c)) were carried out. Here the sensor response is again due to both residual gases and $\mathrm{CO}$ with different concentration. The response to the residual gases during the whole measurement was also determined by setting the $\mathrm{CO}$ concentration to 0 at the end of cycle 1 and 3. The pure CO response resp $p_{C O}$ has been calculated at different $\mathrm{CO}$ concentrations using (9), and is 
shown in Fig. 4 (d). The CO response increases with the increasing CO concentration from 120 ppb up to 40 ppm indicating that $\mathrm{CO}$ at ppb level is detectable at room temperature, which is also an evidence of the oxidizing effect of $\mathrm{CO}$ at the photostimulated $\mathrm{ZnO}$ nanowire surface at room temperature. Furthermore, although the resistance change by exposure to $\mathrm{CO} \&$ residual gases, i. e. resp $p_{C O \text { \&res }}$, in the former cycles is higher than that in the latter cycles, the calculated pure CO response, however, remained nearly the same for the same $\mathrm{CO}$ concentration in the different cycles (Fig. 4 (d)), indicating that the sensing signal is quite reproducible, which is very important for real applications.

\subsection{CO Sensing Mechanism}

The mechanism of $\mathrm{CO}$ adsorption on $\mathrm{ZnO}$ has been widely studied both theoretically and experimentally on a variety of facets with different polarity (polar or non-polar) and different structural properties (single crystalline, defect-rich and highly doped). Using density-functional theory, it was determined that CO can adsorb

on both polar (Zn-terminated (0001)) and non-polar (10 $\overline{1} 0)$ surfaces, with the C-atoms bound not to O-ions but $\mathrm{Zn}$ ions, in particular on step edges and other defects [32]. Photoelectron spectroscopy measurements revealed that $\mathrm{CO}$ adsorbs on (0001), (000 $\overline{1})$ polar and $(10 \overline{1} 0),(11 \overline{2} 0)$ non-polar surfaces at surface defects, in particular oxygen vacancies at steps [27].

In the case of $\mathrm{CO}$ adsorption on photostimulated $\mathrm{ZnO}$ nanowires, because gas molecules can adsorb both at the surface and on the side wall of $\mathrm{ZnO}$ nanowires, $\mathrm{CO}$ adsorption on both polar and non-polar $\mathrm{ZnO}$ surfaces is possible. There are abundant of wires with a huge surface with defects [33]. Thus, defects including oxygen vacancies are pre-existing on the surface of $\mathrm{ZnO}$ nanowires in the initial state [34]. In air, the oxygen vacancies are compensated by chemisorbed $\mathrm{O}_{2}^{-}$at room temperature which leads to a reduction of free electron concentration and high resistivity of the nanowires, as determined electrically. After photostimulation, the charged adsorbed oxygen species partially desorbs leaving more defects, mostly non-compensated oxygen vacancies $V_{O}$ at the surface. As a result, free electrons are released and the nanowire resistivity decreases. On the other hand, the amount of photo-generated electrons in the core of the $\mathrm{ZnO}$ nanowires is relatively small due to the low intensity of the UV light. Thus, photoelectrons have only negligible contribution to the density of free carriers and the large resistance change is basically a surface effect. The corresponding chemical reaction during this process can be described as follows [35]: 
$\mathrm{O}_{2}^{-} \stackrel{\mathrm{hv}}{\longrightarrow} \mathrm{O}_{2}+\mathrm{V}_{\mathrm{O}}+\mathrm{e}^{-}$

When $\mathrm{CO}$ and residual gases approach the surface, the $\mathrm{CO}$ or adsorbed oxygen molecules do not have enough thermal energy to recombine with oxygen vacancies at room temperature, which is reasonable due to the fact that even at high temperatures above $1000 \mathrm{~K}$, the oxygen vacancies cannot be completely annihilated in an oxygen environment [27]. Thus, a possible mechanism is that the ionized $\mathrm{CO}$ molecules adsorb at the nanowire surface with the C-atoms weakly bonded to $\mathrm{Zn}$ ions at surface defects (oxygen vacancies) at room temperature [32], leading to a decrease of surface electron density. Thus, the resistance of $\mathrm{ZnO}$ nanowires increases after $\mathrm{CO}$ exposure. However, further experiments are still desired to understand the dipole nature of the $\mathrm{CO}$ molecules adsorbed on the surface.

\section{Conclusion}

In conclusion, it has been demonstrated that $\mathrm{CO}$ can act as an oxidizing gas on photostimulated $\mathrm{ZnO}$ nanowires at room temperature. UV irradiation can effectively desorb charged adsorbates from the surface, which releases free electrons. After $\mathrm{CO}$ exposure, $\mathrm{CO}$ molecules adsorb at the surface of $\mathrm{ZnO}$ nanowires, trapping free electrons and leading to a decrease of surface conductivity. The oxidizing behavior of $\mathrm{CO}$ by means of photostimulation opens the way for room temperature $\mathrm{CO}$ detection, in particular in fuel cells containing $\mathrm{CO}$ as a rest gas in $\mathrm{H}_{2}$, while for $\mathrm{CO}$ detection in $\mathrm{H}_{2} \mathrm{O}$ and $\mathrm{O}_{2}$ containing environment, response to residual gases needs to be determined for the calculation of pure CO response. These results shed light on developing roomtemperature gas sensors using oxide nanostructures with the help of high energetic photon replacing the high temperature process normally used in traditional gas sensors. Furthermore, the $\mathrm{ZnO}$ nanowires can be directly integrated with UV LEDs, leading to compact miniaturized energy-saving CO sensors [28].

\section{Acknowledgment}

This work was supported by the Fraunhofer Research Grants "Attract" and "Challenge". The authors thank Crenguta-Columbina Leancu for the PL measurements. Furthermore, we thank B. Raynor for critically reading the manuscript. S. K. Youn acknowledges DIP-K.6.1 and ZA 191/17-3 for financial support. N. Ramgir would like to thank Humboldt Foundation for the fellowship.

\section{References}

[1] Xia Y N 2008 Nature Mater. 7758 
[2] Hsueh T J, Chen Y W, Chang S J, Wang S F, Hsu C L, Lin Y R, Lin T S and Chen I C 2007 Sens. Actuators B $\mathbf{1 2 5} 498$

[3] Wang X H, Zhang J and Zhu Z Q 2006 Appl. Surf. Sci. 2522404

[4] Tien L C, Wang H T, Kang B S, Ren F, Sadik P W, Norton D P, Pearton S J and Lin J S 2005 Electrochem. Solid-State Lett. 8 G230

[5] Wang C Y, Ali M, Kups T, Rohlig C C, Cimalla V, Stauden T and Ambacher O 2008 Sens. Actuators B 130589

[6] Li L M, Li C C, Zhang J, Du Z F, Zou B S, Yu H C, Wang Y G and Wang T H 2007 Nanotechnology 18225504

[7] Chang J F, Kuo H H, Leu I C and Hon M H 2002 Sens. Actuators B 84258

[8] Liao L, Lu H B, Li J C, Liu C, Fu D J and Liu Y L 2007 Appl. Phys. Lett. 91173110

[9] Zuruzi A S, Kolmakov A, MacDonald N C and Moskovits M 2006 Appl. Phys. Lett. 88102904

[10] Cantalini C, Post M, Buso D, Guglielmi A and Martucci A 2005 Sens. Actuators B 108184

[11] Cruccolini A, Narducci R and Palombari R 2004 Sens. Actuators B 98227

[12] Miura N, Raisen T, Lu G and Yamazoe N 1998 Sens. Actuators B 4784

[13] Ryu H W, Park B S, Akbar S A, Lee W S, Hong K J, Seo Y J, Shin D C, Park J S and Choi G P 2003 Sens. Actuators B 96717

[14] Youn S K, Ramgir N, Wang C Y, Subannajui K, Cimalla V and Zacharias M 2010 J. Phys. Chem. C 11410092

[15] Prades J D, Jimenez-Diaz R, Hernandez-Ramirez F, Barth S, Cirera A, Romano-Rodriguez A, Mathur S and Monante J R 2009 Sens. Actuators B 140 337, Wang C Y, Becker R W, Passow T, Pletschen W, Köhler K, Cimalla V and Ambacher O 2011 Sens. Actuators B 152235

[16] Wang C Y, Cimalla V, Kups T, Rohlig C C, Romanus H, Lebedev V, Pezoldt J, Stauden T and Ambacher O 2007 J. Appl. Phys. 102044310

[17] Youn S K, Ramgir N, Wang C Y, Subannajui K, Cimalla V, Ambacher O and Zacharias M J. Phys. Chem. $C$ In press

[18] Ahn C H, Mohanta S K, Lee N E and Cho H K 2009 Appl. Phys. Lett. 94261904

[19] Djurisic A B and Leung Y H 2006 Small 2944

[20] Liu X, Wu X H, Cao H and Chang R P H 2004 J. Appl. Phys. 953141

[21] Lin B X, Fu Z X and Jia Y B 2001 Appl. Phys. Lett. 79943

[22] Yang Q, Tang K, Zuo J and Qian Y 2004 Appl. Phys. A - Mater. 791847

[23] Shi W S, Cheng B, Zhang L and Samulski E T 2005 J. Appl. Phys. 98083502

[24] Guo B, Qiu Z R and Wong K S 2003 Appl. Phys. Lett. 822290

[25] Iwakami S, Machida O, Yanagihara M, Ehara T, Kaneko N, Goto H and Iwabuchi A 2007 Jpn. J. Appl. Phys. 46 L587

[26] Gay R R, Nodine M H, Henrich V E, Zeiger H J and Solomon E I 1980 J. Am. Chem. Soc. 102 6752

[27] Lindsay R, Michelangeli E, Daniels B G, Ashworth T V, Limb A J, Thornton G, Gutierrez-Sosa A, Baraldi A, Larciprete R and Lizzit S 2002 J. Am. Chem. Soc. 1247117

[28] Wang C Y, Cimalla V, Kups T, Röhlig C C, Stauden T, Ambacher O, Kunzer M, Passow T, Schirmacher W, Pletschen W, Köhler K and Wagner J 2007 Appl. Phys. Lett. 91103509

[29] Keem K, Kim H, Kim G T, Lee J S, Min B, Cho K, Sung M Y and Kim S 2004 Appl. Phys. Lett. 844376

[30] Woll C 2007 Prog. Surf. Sci. 8255

[31] Zhdanov V P 2002 Surf. Sci. 500966

[32] Meyer B and Marx D 2003 J. Phys. - Condens. Mat. 15 L89

[33] Wang D F and Zhang T J 2009 Solid State Communications 1491947

[34] Li Q H, Gao T, Wang Y G and Wang T H 2005 Appl. Phys. Lett. 86123117

[35] Janotti A and Van de Walle C G 2005 Appl. Phys. Lett. 87122102 
Figure captions:

Figure 1. SEM image taken at $45^{\circ}$ tilt of $\mathrm{ZnO}$ nanowires grown on an Au-patterned $\mathrm{Si}$ substrate. The inset shows a schematic view of $\mathrm{ZnO}$ nanowire bridges.

Figure 2. (a) PL measurements using different exciting sources and excitation power densities: solid lines represent the excitation by a HeCd laser $(\lambda \sim 325 \mathrm{~nm})$ and dashed lines by a UV LED $(\lambda \sim 356 \mathrm{~nm})$; (b) PL intensity and intensity ratio between the green and UV emission as a function of excitation power density.

Figure 3. Resistance change of the $\mathrm{ZnO}$ nanowires under photostimulation (black) and photostimulation/CO exposure (red). The inset shows the resistance change after four cycles of photostimulation/CO exposure.

Figure 4. (a) Resistance change of $\mathrm{ZnO}$ nanowires periodically exposed to residual gases (mainly $\mathrm{H}_{2} \mathrm{O}$ and $\mathrm{O}_{2}$ ) and 40 ppm CO in $\mathrm{N}_{2}$; (b) measured nanowire response from (a) and calculated response towards $40 \mathrm{ppm} \mathrm{CO}$ in dependence on the photostimulation/oxidation cycles, (c) resistance change of $\mathrm{ZnO}$ nanowires in $\mathrm{CO}$ with different concentration, and (d) $\mathrm{ZnO}$ nanowire response in dependence on the $\mathrm{CO}$ concentration. 